\title{
Evidências científicas da assistência de enfermagem na realização do exame
}

\section{Papanicolau}

\author{
Scientific evidence of nursing care in the performance of the pap smear \\ Evidencia científica de los cuidados de enfermería en la realización del Papanicolaou
}

Recebido: 28/04/2021 | Revisado: 05/05/2021 | Aceito: 06/05/2021 | Publicado: 21/05/2021

Isa Eduarda de Sousa Rocha

ORCID: https://orcid.org/0000-0002-9951-8349 Centro Universitário Santo Agostinho, Brasil

E-mail: isaeduarda76@gmail.com

Viviane Fortes da Silva

ORCID: https://orcid.org/0000-0002-1449-4908 Centro Universitário Santo Agostinho, Brasil E-mail:vivianefortes32@hotmail.com

Tatiana Maria Melo Guimarães

ORCID: https://orcid.org/0000-0002-2748-6771

Centro Universitário Santo Agostinho, Brasil

E-mail: enfermeiratatianamelo@gmail.com

\begin{abstract}
Resumo
Objetivou-se analisar as evidências cientificas a respeito da assistência de enfermagem no acolhimento das mulheres e na realização do exame Papanicolau. Realizou-se um estudo de revisão integrativa de literatura. Para a busca de dados foram utilizadas fontes de coleta nas plataformas online de pesquisa, SciELO (Scientific Eletronic Library Online), BVS (Biblioteca Virtual em Saúde), LILACS (Literatura Latino Americana em Ciências da Saúde), MEDLINE (Medical Literature Analysis and Retrieval System Online) no período de 2016 a 2020. Foram utilizados os descritores "Diagnóstico Precoce", "Enfermagem", "Exame Papanicolau". Foram selecionados 2.539 artigos ao todo, logo após foram aplicados os critérios de inclusão e selecionados 58 artigos para leitura de títulos e resumos. Após isso, foram selecionados 12 artigos que atendiam ao objetivo da pesquisa para compor o trabalho. Os resultados do estudo evidenciaram que a atuação do enfermeiro é muito importante na prevenção do câncer do colo do útero, visto que a atenção básica acolhe o paciente desde o acolhimento e até o possível diagnóstico, por meio da realização da coleta de material para exame de citologia, visto que o enfermeiro mantém um dos maiores vínculos com as pacientes, conhecer a realidade local da área onde atua é uma das principais estratégias para melhorar o planejamento de ações para reduzir as taxas de morbimortalidade. Concluiu-se que há uma necessidade de uma assistência qualificada, assim como busca ativa, visitas domiciliares, identificação das barreiras para a realização do exame citopatológico, conscientizando as mulheres da importância do diagnóstico precoce.
\end{abstract}

Palavras-chave: Diagnóstico precoce; Enfermagem; Exame Papanicolau.

\begin{abstract}
The objective was to analyze the scientific evidence regarding nursing care in the reception of women and the performance of the Papanicolau exam. An integrative literature review study was conducted. For the search for data, the online research platforms SciELO (Scientific Eletronic Library Online), BVS (Virtual Health Library), LILACS (Latin American Literature on Health Sciences), MEDLINE (Medical Literature Analysis and Retrieval System Online) were used in the period from 2016 to 2020. The descriptors "Early Diagnosis", "Nursing", "Pap smear" were used. The total of 2,539 articles were selected, soon after, the inclusion criteria were applied and 58 articles were selected for reading titles and abstracts. After that, 12 articles that met the research objective were selected to compose the work. The results of the study showed that the role of nurses is very important in the prevention of cervical cancer, since primary care welcomes patients from the reception to the possible diagnosis, through the collection of material for cytological examination, since nurses maintain one of the strongest links with patients, Knowing the local reality of the area where they work is one of the main strategies to improve the planning of actions to reduce morbidity and mortality rates. It was concluded that there is a need for qualified assistance, as well as active search, home visits, identification of barriers to cytopathological examination, making women aware of the importance of early diagnosis.
\end{abstract}

Keywords: Early diagnosis; Nursing; Pap smear. 


\begin{abstract}
Resumen
El objetivo fue analizar la evidencia científica sobre el cuidado de enfermería en la acogida de mujeres y la realización del Papanicolaou. Se realizó un estudio de revisión integradora de la literatura. Para la búsqueda de datos se utilizaron fuentes de recolección en las plataformas de investigación en línea, SciELO (Scientific Electronic Library Online), BVS (Virtual Health Library), LILACS (Latin American Literature in Health Sciences), MEDLINE (Medical Literature Analysis and Retrieval System Online), en el período de 2016 a 2020. Se utilizaron los descriptores "Diagnóstico precoz", "Enfermería", "Examen de Papanicolaou". Se seleccionaron un total de 2.539 artículos, poco después de que se aplicaron los criterios de inclusión y se seleccionaron 58 artículos para leer títulos y resúmenes. Posteriormente, se seleccionaron 12 artículos que cumplieron con el objetivo de investigación para componer el trabajo. Los resultados del estudio mostraron que la actuación de la enfermera es muy importante en la prevención del cáncer de cérvix, ya que la atención primaria acoge a la paciente desde el momento de la recepción y hasta el posible diagnóstico, a través de la recolección de material para examinar la citología, ya que las enfermeras mantienen uno de los mayores vínculos con los pacientes, conocer la realidad local del área donde se trabaja es una de las principales estrategias para mejorar la planificación de acciones para reducir las tasas de morbilidad y mortalidad Se concluyó que existe una necesidad de asistencia calificada, así como búsqueda activa, visitas domiciliarias, identificación de barreras para realizar examen citopatológico, concienciando a las mujeres de la importancia del diagnóstico precoz.

Palabras clave: Diagnóstico precoz; Enfermería; Prueba de Papanicolaou.
\end{abstract}

\title{
1. Introdução
}

O câncer do colo do útero é um importante problema de saúde pública em países em desenvolvimento, chegando a ser um dos tipos mais comum na população feminina. Comparando às outras neoplasias, é altamente prevenível, apresenta evolução lenta até atingir o estágio de câncer invasivo e dispõe de exame de rastreamento tecnicamente simples e eficaz na sua detecção (Soares; Silva, 2016). É uma doença que pode seguir sem nenhum sintoma em fase inicial e evoluir para quadros de sangramento vaginal contínuo ou após a relação sexual, secreção vaginal anormal e dor abdominal associada com queixas urinárias ou intestinais em casos mais avançados (brasil, 2016).

A mortalidade segundo o INCA (2017), aumenta progressivamente após os quarenta anos de idade com grande diferença entre as regiões brasileiras. No Brasil em 2018, ocorreram seis mil quinhentos e vinte e seis óbitos por esta neoplasia, representando uma taxa ajustada de mortalidade por este câncer de 6,10/100 mil mulheres (Inca, 2020). O colo do útero apresenta uma parte interna, que constitui o canal cervical ou endocérvice, que é revestido por uma camada única de células cilíndricas produtoras de muco. A parte externa, que mantém contato com a vagina, é chamada de ectocérvice e é revestida por um tecido de várias camadas de células planas (Brasil, 2016).

O Ministério da Saúde ainda afirma que para rastrear o câncer do colo do útero e suas lesões percursoras deve ser realizado o exame citopatológico. Tal exame possui um intervalo de realização de três anos em caso dois exames anteriores deem negativos, com intervalo anual (Brasil, 2016). Deve-se analisar o rastreamento do câncer de colo de útero a partir das características da coleta dos exames, segundo Ceolin et al., (2020), para que possam detectar as células precoces com características alteradas e, com isso, serem efetivos no rastreamento do câncer.

A coleta deve ter início aos 25 anos de idade para mulheres que possuem atividade sexual, com seguimento até os 64 anos de idade. Após essa idade, o rastreamento deverá ser encerrado, se pelo menos dois exames com resultado negativo realizados dentro dos últimos cinco anos. Caso a mulher possua sessenta e quatro anos e nunca tenha realizado exame preventivo, deverá realizar dois exames com intervalos de um até três ano, sendo resultados negativos, estas estarão dispensadas do exame citopatológico. O exame de Papanicolau é oferecido pelo Sistema Único de Saúde (SUS) na Atenção Primária à Saúde por meio das equipes de saúde da família. (Brasil, 2016).

A consulta de enfermagem é uma atividade privativa do enfermeiro, com respaldo legal desde 1986, que consiste no desenvolvimento de ações deliberadas e sistematizadas, tendo como principal objetivo promover o cuidado. Consiste em um instrumento de trabalho para acompanhar mudanças no estilo de vida e detectar necessidades de intervenção ou acompanhamento, e buscando ações de desenvolvimento e fortalecimento para o autocuidado, por meio do Processo de 
Enfermagem (Moreira et al., 2012). Portanto, para qualificar a assistência, a coleta do exame citopatológico do colo do útero deve ocorrer no âmbito da consulta de enfermagem à mulher (Cofen, 2011)

Nesse contexto, o estudo se justifica através da importância de uma boa assistência de enfermagem na saúde da mulher enfatizada pela prevenção do câncer de colo uterino, além de analisar os conhecimentos das mulheres a respeito do assunto evidenciadas nos estudos científicos. Desta forma, este estudo contribui tanto para o conhecimento acadêmico como para os profissionais que trabalham nas áreas da saúde, além de servir de subsídio para implementação de estratégias na Atenção Básica à saúde.

Assim, faz-se necessário a busca de mais estudos para que possam proporcionar uma melhor assistência, atenção e preocupação das equipes de saúde multiprofissionais para acompanhar e identificar as necessidades individuais durante a realização do exame no quesito de descoberta do câncer de colo de útero. Nesta perspectiva, a pesquisa tem como questão norteadora: Quais as evidências científicas abordam a assistência de enfermagem na prevenção do câncer de colo de útero? Como objetivo do estudo: descrever as evidências cientificas a respeito da assistência de enfermagem no acolhimento das mulheres e na realização do exame Papanicolau.

\section{Metodologia}

Realizou-se uma pesquisa de revisão integrativa de literatura, para identificar e descrever as evidências sobre o câncer do colo de útero e a assistência de enfermagem frente a realização do exame Papanicolau, fatores que interferem na realização do exame, baseado em evidências cientificas.

A revisão integrativa é uma abordagem metodológica que se refere às revisões o qual permite uma inclusão de estudos não experimentais e experimentais para serem compreendidos e analisados, proporcionando uma síntese de conhecimentos, através de resultados de estudos significativos na prática. No processo de elaboração apresentam-se seis fases: elaboração da pergunta norteadora, amostragem ou busca na literatura, coleta de dados, análise crítica dos estudos incluídos, discussão de resultados e apresentação da revisão (Souza et al., 2010).

A primeira etapa do processo deve ser norteadora para a construção de um trabalho bem elaborado estando relacionada a um raciocínio teórico e incluindo definições já aprendidas assim a definição do tema será de forma clara e específica. A segunda etapa determina que quanto mais amplo for o objetivo da revisão mais seletivo deverá ser a revisão quanto à inclusão da pesquisa a ser estudada. Na terceira etapa deve se realiza a organização e formação do banco de dados. Já na quarta etapa a organização do revisor contribui na avaliação crítica dos estudos e na tomada de decisão para a utilização dos resultados de pesquisas. A quinta etapa realiza se a comparação dos conhecimentos e na sexta e última etapa contém a descrição das etapas e os principais resultados evidenciados da análise dos artigos incluídos (Mendes, Silveira \& Galvão, 2008).

O levantamento de dados foi realizado nas bases de dados das plataformas online de pesquisa, SciELO (Scientific Eletronic Library Online), LILACS (Literatura Latino Americana em Ciências da Saúde), MEDLINE (Medical Literature Analysis and Retrieval System Online). Foram utilizados como descritores: "Diagnóstico Precoce"; "Enfermagem"; "Exame Papanicolau". Como estratégia de busca foi realizada o cruzamento dos descritores com o operador booleano "AND": "Diagnóstico precoce AND Enfermagem; "Diagnóstico precoce AND Exame Papanicolau"; "Enfermagem AND Exame Papanicolau".

Como critérios de inclusão foram selecionados artigos publicados em periódicos nacionais e internacionais, disponibilizados na íntegra (texto completo) em língua portuguesa, inglesa, e espanhola que abordem o tema principal proposto, câncer de colo de útero, no período de 2016 a 2020. Foram excluídos os artigos incompletos, dissertações, como 
publicações anteriores ao ano de 2016, sem relevância científica, os que se apresentarem de forma repetida e estudos de revisão bibliográfica.

Após a busca nas bases de dados obteve se um total de 2.539 artigos. Em seguida, depois da leitura dos títulos, foi notado que nem todos possuíam os critérios para entrar para o estudo, e foram selecionados 58 artigos para leitura dos resumos, e excluídos os que não abordavam o propósito da pesquisa. Após a leitura foram selecionados 12 artigos para análise e categorização, conforme a Figura 1 apresenta.

Figura 1: Fluxograma PRISMA (Moher, et al., 2015). Representativo do processo de revisão, Teresina-PI, 2021.
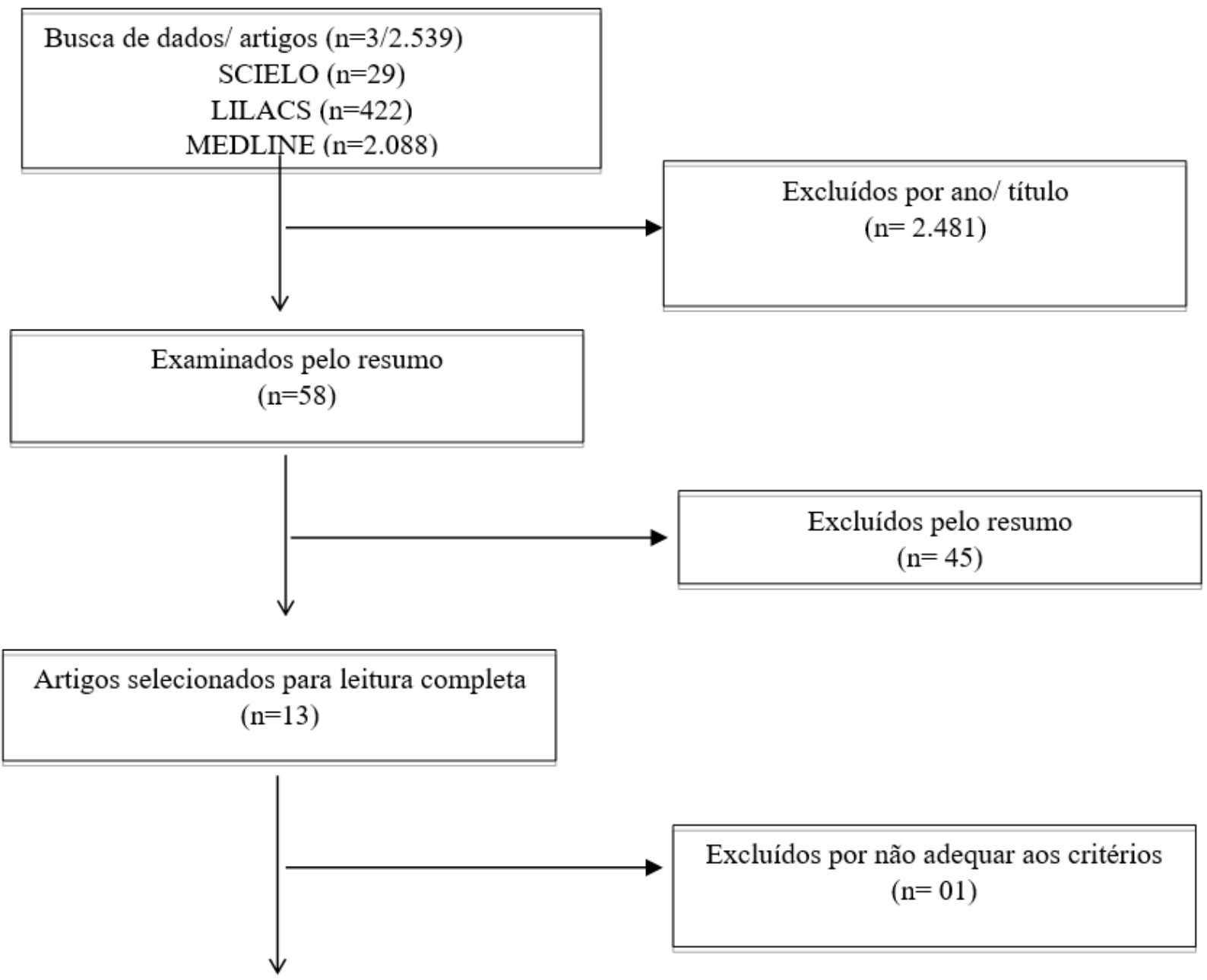

\section{Artigos incluídos na revisão integrativa} $(\mathrm{n}=12)$

Fonte: Coleta de dados, Teresina-PI (2021).

A análise dos artigos selecionados foi realizada por meio de uma leitura criteriosa e rigorosa, seguindo todos os critérios escolhidos previamente. A caracterização foi realizada através de um formulário, onde favoreceu a organização, e a extração de todas as informações relevantes ao estudo, os objetivos propostos e os resultados em evidências 


\section{Resultados}

Após a análise de todo o material selecionado, as informações foram organizadas e agrupadas inicialmente em quadros, para uma melhor distribuição e de fácil compreensão para leitura. A Tabela 1 mostra os estudos selecionados segundo ano de publicação, abordagem metodológica, e idioma.

Tabela 1: Distribuição dos artigos conforme idioma, ano de publicação, e abordagem metodológica. Teresina, 2021.

\begin{tabular}{lcc}
\hline VARIÁVEIS & $\mathbf{N}^{\mathbf{0}}$ & \% \\
\hline ANO DE PUBLICAÇÃO & & 33,33 \\
2016 & 4 & 8,33 \\
2017 & 1 & 25,0 \\
2019 & 3 & 33,33 \\
2020 & 4 & \\
ABORDAGEM METODOLÓGICA & & 58,33 \\
Quantitativo & 7 & 41,66 \\
Qualitativo & 5 & \\
IDIOMA & & 50,0 \\
Português & 6 & 50,0 \\
Inglês & 6 & \\
\hline
\end{tabular}

Fonte: Pesquisa direta em base de dados, Teresina (2021).

De acordo com a apresentação dos resultados dos dados avaliados após o levantamento bibliográfico, foi observado, conforme a Tabela 1, que os anos com maiores publicações foram $2016(33,33 \%)$ e $2020(33,33 \%)$.

Em relação a abordagem metodológica, predominou-se a quantitativa com $(58,33 \%)$, e a qualitativa com $(41,66 \%)$. Uma abordagem quantitativa possui a sua relevância quando permite unir dados de forma objetiva através das estatísticas que se obtém de informações precisas. A abordagem qualitativa possui sua relevância uma vez que enfatiza de caráter subjetivo o objeto analisado, o qual estuda-se as suas particularidades e as experiências do indivíduo. Em comparação aos idiomas das publicações verificou-se a predominância da língua portuguesa (50\%) e da língua inglesa (50\%).

Analisou-se 12 artigos científicos lidos na integra e divididos em um quadro de forma resumida, abordando as variáveis: título, ano, autores e evidências (Quadro 1). Os artigos foram enumerados de I a XII com a finalidade de melhor visualização da análise.

Quadro 1: Distribuição dos estudos conforme o título, ano, autores e evidências. Teresina, 2021.

\begin{tabular}{|c|c|c|c|c|}
\hline $\mathbf{N}^{\circ}$ & TÍTULO & ANO & AUTORES & EVIDÊNCIAS \\
\hline I & $\begin{array}{l}\text { Desigualdades } \\
\text { socioeconômicas e regionais } \\
\text { na cobertura de exames } \\
\text { citopatológicos do colo do } \\
\text { útero. }\end{array}$ & 2016 & $\begin{array}{l}\text { Manica, ST; Drachler, } \\
\text { MDL; Teixeira, LB; } \\
\text { Ferla, AA; Gouveia, } \\
\text { HG; Anschau, F; } \\
\text { Oliveira, DLLC. }\end{array}$ & $\begin{array}{l}\text { A distribuição das mulheres de } 25 \text { a } 64 \text { anos de idade, no } \\
\text { período de 2011-2012, conforme a vulnerabilidade social } \\
\text { municipal e as macrorregiões do Estado, mostra que } 78,1 \% \\
\text { dessa população residiam em municípios com } \\
\text { vulnerabilidade social relativamente baixa. }\end{array}$ \\
\hline II & $\begin{array}{l}\text { Social network characteristics } \\
\text { and cervical câncer screening } \\
\text { among Quechua women in } \\
\text { Andean Peru }\end{array}$ & 2016 & $\begin{array}{l}\text { Luque, JS.; Opoku, } \\
\text { S.; Ferris, DG.; } \\
\text { Guevara, WS.; }\end{array}$ & $\begin{array}{l}\text { Neste estudo observou se que as vizinhas mais podem ter } \\
\text { fornecido uma importante fonte de apoio social para } \\
\text { decisões relacionadas à saúde relacionadas ao recebimento } \\
\text { de um exame de Papanicolau e que mais estudos devem } \\
\text { coletar dados para medir os efeitos da rede de intervenções } \\
\text { de rastreamento. }\end{array}$ \\
\hline
\end{tabular}




\begin{tabular}{|c|c|c|c|c|}
\hline III & $\begin{array}{l}\text { Muslim immigrant women's } \\
\text { views on cervical cancers } \\
\text { creening and HPV self- } \\
\text { sampling in Ontario, Canada }\end{array}$ & 2016 & $\begin{array}{l}\text { Vahabi, M.; Lofters, } \\
\text { A. }\end{array}$ & $\begin{array}{l}\text { Este estudo fornece insights críticos sobre a importância } \\
\text { das crenças religiosas e culturais na formação do cotidiano } \\
\text { e o impacto dissuasor das crenças e práticas de saúde nos } \\
\text { países de origem na utilização de serviços de rastreamento } \\
\text { por mulheres imigrantes muçulmanas. }\end{array}$ \\
\hline IV & $\begin{array}{l}\text { Impact of variations in triage } \\
\text { cytology interpretation } \\
\text { on human papillomavirus- } \\
\text { based cervical screening } \\
\text { and implications for screening } \\
\text { algorithms }\end{array}$ & 2016 & $\begin{array}{lr}\text { Ronco, G; Zappa, } & \text { M.; } \\
\text { Franceschi, } & \text { S.; } \\
\text { Tunesi, } & \text { S.; } \\
\text { Caprioglio, } & \text { A.; } \\
\text { Confortini, } & \text { M.; } \\
\text { Mistro, AD.; Carozzi, } & \text { F.; Segnan, N.; Zorzi, } \\
\text { M.; }\end{array}$ & $\begin{array}{l}\text { O estudo mostra a proporção de mulheres com HPV } \\
\text { diretamente encaminhadas para colposcopia; no geral, 63\% } \\
\text { dos HPV foram encaminhados para colposcopia } \\
\text { imediatamente ou na repetição do HPV. Um aumento } \\
\text { absoluto de } 10 \% \text { no encaminhamento imediato da } \\
\text { colposcopia resultou em } 4,2 \% \text {. Aumento no } \\
\text { encaminhamento geral. Um aumento absoluto de } 10 \% \text { na } \\
\text { sensibilidade da citologia. }\end{array}$ \\
\hline $\mathbf{V}$ & $\begin{array}{l}\text { Screening for cervical cancer } \\
\text { in imprisoned women in Brazil }\end{array}$ & 2017 & $\begin{array}{l}\text { Silva, ERPD.Souza, } \\
\text { ASD.; Souza, TGBD.; } \\
\text { Tsuha, DH.; Barbieri, } \\
\text { AR.; }\end{array}$ & $\begin{array}{l}\text { O estudo mostra que a maioria das presidiárias tinha } \\
\text { educação limitada, usava tabaco e tinha fatores de risco } \\
\text { fundamentais para o desenvolvimento de câncer de colo do } \\
\text { útero. }\end{array}$ \\
\hline VI & $\begin{array}{l}\text { Insegurança nas ações de } \\
\text { controle do câncer de colo } \\
\text { uterino: atuação do enfermeiro } \\
\text { na Estratégia de Saúde da } \\
\text { Família. }\end{array}$ & 2019 & $\begin{array}{l}\text { Rocha, CBAR; Cruz, } \\
\text { JW; Oliveira, JCS. }\end{array}$ & $\begin{array}{l}\text { O estudo revelou a insegurança por parte dos enfermeiros } \\
\text { na realização do exame Papanicolau, decorrente da falta de } \\
\text { experiência, da insuficiência de conhecimento fornecido na } \\
\text { graduação, da necessidade do auxílio de outro profissional } \\
\text { para tomada de decisão e da carência de capacitações }\end{array}$ \\
\hline VII & $\begin{array}{l}\text { Effectiveness of cervical } \\
\text { screening after age } 60 \text { years } \\
\text { according to screening history: } \\
\text { nationwide cohort study in } \\
\text { sweden }\end{array}$ & 2019 & $\begin{array}{l}\text { Wang, J.; Andrae, B.; } \\
\text { Sundstro, K.; Ploner, } \\
\text { A.; Stro, P.; Elfstro, } \\
\text { M.; Dillner, J.; }\end{array}$ & $\begin{array}{l}\text { Neste estudo, o rastreamento cervical com citologia aos } 61- \\
65 \text { anos foi associado a uma redução estatisticamente do } \\
\text { risco de câncer do colo do útero para mulheres que não } \\
\text { foram triadas ou rastreadas com anormalidades na casa dos } \\
50 \text { anos. Em mulheres rastreadas com resultados normais } \\
\text { na década de } 50 \text {, o risco de câncer futuro não foi } \\
\text { considerável; }\end{array}$ \\
\hline VIII & $\begin{array}{l}\text { Cervical cancer: knowledge, } \\
\text { attitude and practice on the } \\
\text { prevention examination }\end{array}$ & 2019 & $\begin{array}{l}\text { Melo, EMFD; } \\
\text { Linhares, MPR; Silva, } \\
\text { TMD; Pontes, CL; } \\
\text { Santos, AHDS; } \\
\text { Oliveira, SCD. }\end{array}$ & $\begin{array}{l}\text { Analisou-se que as mulheres presentes no estudo conhecem } \\
\text { a necessidade da realização do exame, porém não } \\
\text { conhecem sobre a importância da realização do mesmo. } \\
\text { Diante disso, visa-se a necessidade das ações educativas } \\
\text { por parte da enfermagem. }\end{array}$ \\
\hline IX & $\begin{array}{l}\text { Exclusividade na coleta de } \\
\text { material para exame de } \\
\text { colpocitologiaoncótica: } \\
\text { percepção dos enfermeiros }\end{array}$ & 2020 & $\begin{array}{l}\text { Oliveira, LL; Santos, } \\
\text { MRS; Rodrigues, } \\
\text { ILA; André, SR; } \\
\text { Silva, IFS; Nogueira, } \\
\text { LMV. }\end{array}$ & $\begin{array}{l}\text { No estudo observou-se as dificuldades relatadas pelos } \\
\text { enfermeiros com implicações diretas na realização do } \\
\text { exame, como a falta de recursos materiais; o espaço físico } \\
\text { inadequado ou compartilhado; a sobrecarga de trabalho e a } \\
\text { coleta realizada por profissionais do sexo masculino }\end{array}$ \\
\hline $\mathbf{X}$ & $\begin{array}{l}\text { Análise dos resultados do } \\
\text { último laudo citopatológico de } \\
\text { pacientes com Papanicolau em } \\
\text { atraso }\end{array}$ & 2020 & $\begin{array}{l}\text { Maciel, NS; Luxia, } \\
\text { FJM; Ferreira, DDS; } \\
\text { Silva, } \quad \text { MCDLP; } \\
\text { Joaquim, DC; Sousa, }\end{array}$ & $\begin{array}{l}\text { Observou-se que possui um número satisfatório de } \\
\text { mulheres com o exame Papanicolau em atraso, como } \\
\text { também uma deficiência na qualidade dos exames, indicada } \\
\text { pela ausência de tecido metaplásico. }\end{array}$ \\
\hline
\end{tabular}




\begin{tabular}{|c|c|c|c|c|}
\hline & & & LBD. & \\
\hline XI & $\begin{array}{l}\text { Exame citopatológico do colo } \\
\text { uterino: descrição dos } \\
\text { principais indicadores em um } \\
\text { município nordestino }\end{array}$ & 2020 & $\begin{array}{l}\text { Rodrigues, } \quad \text { M.; } \\
\text { Moraes, M. }\end{array}$ & $\begin{array}{l}\text { Os dados mostraram que resultados sugestivos de alto grau } \\
\text { de malignidade existem em menor frequência nos exames } \\
\text { preventivos e necessitam ser avaliados por exames mais } \\
\text { invasivos. }\end{array}$ \\
\hline XII & $\begin{array}{l}\text { Estratégias Utilizadas para } \\
\text { Melhorar a Qualidade dos } \\
\text { Exames Citopatológicos }\end{array}$ & 2020 & $\begin{array}{l}\text { Santos, MJS; Ribeiro, } \\
\text { AA. }\end{array}$ & $\begin{array}{l}\text { O estudo revelou que de } 6.809 \text { diagnósticos, observaram-se } \\
99,4 \% \text { satisfatórios, sendo } 91,3 \% \text { resultados negativos, } \\
8,1 \% \text { diagnósticos com anormalidades citológicas e } 0,6 \% \\
\text { de exames insatisfatórios. Portanto, é importante a } \\
\text { observação do profissional durante toda a realização do } \\
\text { exame. }\end{array}$ \\
\hline
\end{tabular}

Fonte: Pesquisa direta em base de dados. Teresina (2021).

Com base nos estudos apresentados salienta-se os principais pontos abordados sobre a Assistência de Enfermagem na prevenção do câncer do colo do útero nos artigos selecionados.

A atuação do enfermeiro é muito relevante na prevenção do câncer do colo do útero, visto que a atenção básica acolhe o paciente desde o acolhimento e até o possível diagnóstico, por meio da realização da coleta de material para exame de citologia (Oliveira, et al., 2020). Dentre os fatores que permitem que o enfermeiro seja capacitado para a detecção precoce/rastreamento do câncer de colo do útero está a qualidade da formação profissional que deve ser contemplada na graduação, contudo fazem-se indispensáveis atualizações de informações complementares baseadas em livros, artigos e manuais do Ministério da Saúde (Rocha, Cruz \& Oliveira, 2019).

Alguns profissionais quando perguntados sobre o conhecimento da resolução COFEN n ${ }^{\circ} 385 / 2011$, cerca de 17 referiram várias fontes de acesso à informação, 9 disseram que foram comunicados pela instituição em que trabalham, apenas 3 por meio do COFEN/COREN e 5 só tiveram conhecimento através de acesso pelas mídias sociais (Oliveira, et al., 2020). Eles relatam estarem seguros quanto à realização do procedimento, entretanto, pode-se inferir que alguns profissionais sentem insegurança quanto à realização do exame Papanicolau, quando é difícil localizar o colo do útero (Rocha et al.,2019).

O enfermeiro mantém um dos maiores vínculos com as pacientes, conhecer a realidade local da área onde atua é uma das principais estratégias para melhorar o planejamento de ações para reduzir as taxas de morbimortalidade (Maciel, et al., 2020). Pacientes relataram que o enfermeiro é o profissional habilitado para a realização do exame preventivo, por se tratar de um procedimento que exige tomada de decisões e orientações voltadas para cada paciente (Oliveira, et al., 2020). Muitas mulheres comentaram que a dificuldade pela falta de conhecimento sobre o serviço de saúde mostra como é difícil encontrar um profissional habilitado para realização de exames preventivos (Vahabi \& Lofters, 2016). Outras afirmaram que as vizinhas mais do que os próprios familiares fornecem uma importante fonte de apoio social para as mulheres em relação as decisões relacionadas à saúde relacionadas a realização do exame preventivo e resultados do exame (Luque, et al., 2016).

Em prisões o rastreamento através do exame citológico não é sistemático e regular e os resultados não representam os números dos casos. Sendo necessário organizar os serviços de saúde dentro desse ambiente prisional, para que sejam feitos os exames preventivos (Silva, et al., 2017). A subjetividade utilizada na interpretação da citologia tem um impacto na vida dos profissionais e mulheres que nem sempre recebem boas notícias (Ronco, et al., 2016). Considerando as mulheres não beneficiárias de planos de saúde elas têm no SUS a principal e única fonte de atenção à saúde (Manica, et al., 2016).

Observou-se ainda que características como ter entre 40 e 59 anos e possuir três ou mais filhos com fatores sociodemográficas desfavorecem as mulheres no acesso à realização do exame preventivo como ainda a baixa escolaridade e a 
baixa renda familiar (Melo, et al., 2019). A história anterior de rastreamento pode ser também outro fator importante na determinação do risco de câncer cervical e do impacto de exames complementares, principalmente em mulheres mais velhas (Wang, et al., 2019).

O exame Papanicolau é reconhecido como uma das estratégias mais eficazes para o rastreio de lesões sugestivas de câncer. Por sua fácil execução e valor de baixo custo favorece a sua alta utilização como método de investigação do câncer de colo de útero nas mulheres (Rodrigues \& Moraes, 2020). A educação continuada dos profissionais que participam das etapas do processo do exame Papanicolau é uma forma de que possíveis erros possam ser corrigidos e medidas preventivas tomadas para uma melhor qualidade na realização e prestação da assistência (Santos \& Ribeiro, 2020).

No Quadro 2 foram organizados os estudos de acordo com o assunto principal destacado em seus conteúdos após a leitura completa dos artigos que serão discutidos no próximo tópico.

Quadro 2: Distribuição dos estudos conforme o foco das evidências apresentadas, Teresina, 2021.

\begin{tabular}{|c|l|}
\hline ARTIGOS & \multicolumn{1}{|c|}{ EVIDÊNCIAS } \\
\hline $4,5,6,9,10,11$ & $\begin{array}{l}\text { Assistências do profissional de enfermagem na prevenção do câncer de colo } \\
\text { uterino e suas dificuldades }\end{array}$ \\
\hline $1,2,3,7,8,12$ & Barreiras para a realização do exame Papanicolau \\
\hline
\end{tabular}

Fonte: Pesquisa direta em bases de dados, Teresina (2021).

De acordo com a organização dos estudos conforme observado acima, foram elaboradas duas categorias para apresentação das evidências e discussão da temática.

\section{Discussão}

\subsection{Assistências do profissional de enfermagem na prevenção do câncer de colo uterino e suas dificuldades}

A Resolução COFEN n ${ }^{\circ}$ 385/2011 apresenta a coleta do Papanicolau como prática de cuidado complexo e que precisa ter conhecimento científico, sendo o profissional enfermeiro capacitado para realização do exame, visto que ele deve ser conhecimentos, competências, e habilidades que garantam rigor ao procedimento, atentando para a capacitação contínua necessária à sua realização (Cofen, 2011). Isso reforça os resultados obtidos através da nossa revisão visto que os profissionais de enfermagem sabem da alta complexidade que envolve a coleta do material para o exame e expressam por meio de afinidade com a execução da pratica.

Para Catafesta et al., (2015) a consulta ginecológica, além de ter como objetivo prestar uma boa assistência deve relacionar os aspectos biológicos, sociais e psicológicos, para que a assistência seja realizada de forma inovadora, integral e transformadora. Fortalecendo as evidencias utilizadas na revisão bibliográfica onde afirma que os profissionais devem sentir-se preparados para oferecer uma assistência que proporcione resultados positivos.

Nos estudos de Fonseca, et al., (2019) no que diz respeito à classificação dos atendimentos realizados na Unidades Básica de Saúde foram classificados como bom e regular; à conduta dos profissionais de saúde diante do resultado do exame, cerca de $94 \%$ das mulheres disseram que os profissionais realizaram orientações em saúde. Este resultado corrobora com a nossa revisão visto que o profissional de saúde deve estar seguro para realizá-lo e para orientar as pacientes, visto que no ato do exame ele é responsável por fornecer informações à mulher, realizar o acolhimento e manter a privacidade na consulta de enfermagem. 
Fernandes e Oliveira (2017) afirmam que as ações e programas governamentais de controle do CCU configuram-se em quatro elementos fundamentais: prevenção primária, detecção precoce, diagnóstico/ tratamento e cuidados paliativos direcionados para mulheres em todos os níveis de atenção, especialmente na atenção primária à saúde. Além disso no estudo de Brandão, Andrade e Olivindo (2020) seus resultados mostraram que com relação as dificuldades e limitações que o enfermeiro encontra no local de trabalho muitas delas são relacionadas a falta de insumos, pouca experiência e capacitação para o rastreamento. Consolidando os resultados do nosso estudo observamos que apontam a necessidade da implementação de busca ativa de pacientes com o exame Papanicolau em atraso, especialmente das que apresentam algumas infecções sexualmente transmissíveis ou alteração celular pré-maligna ou maligna.

O estudo de Baia et al., (2018), afirma que muitas mulheres continuam na resistência em realizar o exame por conceitos e valores culturais absorvidos por toda a vida, a falta de informação e vergonha de se expor. Onde se fortalece no nosso estudo através da importância do incentivo a programas de capacitação para os profissionais da saúde, principalmente sobre à captação de mulheres para rastreio e avaliação dos resultados dos exames e ao seguimento das pacientes rastreadas com câncer de colo de útero.

\subsection{Barreiras para a realização do exame Papanicolau}

Manica et al., 2016, afirmam no seu estudo que no Rio Grande do Sul, a cobertura de exame citopatológico do colo de útero foi em média 17,3\% para o total de mulheres residentes e 23,8\% entre mulheres não beneficiárias de planos privados de saúde. Portanto, esse estudo nos mostra que o SUS (Sistema Único de Saúde) ainda é a única opção de assistência em saúde, devido a grande maioria da população não possuir renda e recursos próprios para financiar o serviço de saúde da rede privada.

Barreto, aponta que as barreiras de acesso ao exame preventivo estão muito relacionadas à visão da necessidade e desejo pelo cuidado, ao receio que alguma mulher tem em realizá-lo por desconhecimento e tabus, imposições misóginas do cônjuge, pudor da exposição do corpo ou, por conta de idade ou gênero do profissional. Por vezes, são presentes em inúmeros estudos e reafirmam a competência cultural dos profissionais como atributo fulcral, por meio do reconhecimento de diferentes necessidades dos grupos populacionais, suas características étnicas, raciais e culturais, a entender suas representações dos processos saúde enfermidade (Barreto, 2017).

Para Luque, et al., (2016), o que leva a não realização do exame preventivo são as limitações de tempo que ocorrem devido aos compromissos familiares, a questão de cuidados das crianças, dentre outros. O estudo de Wang et al., (2017), apontou que mulheres idosas na faixa dos 50 anos muitas vezes não realizam o exame preventivo e não são rastreadas por não terem como ir realizar o exame e enfrentam um risco alto de câncer. Essa revisão corroba para uma necessidade muito grande de enfatizar a importância da realização do rastreamento do câncer de colo de útero, visto que tratado precoce o risco do câncer se proliferar é menor.

Já nos estudos de Vahabi e Lofters (2016), é informado que no Canadá as crenças culturais e religiosas possuem uma influência considerável no comportamento de saúde dos indivíduos, pois elas afetam a opinião das mulheres sobre o câncer tendo assim como uma sentença devido a disposição de revelar o histórico familiar de câncer, pois ainda era um tabu em torno da sexualidade feminina e das atividades sexuais. Percebe-se nesse estudo que ainda há uma resistência das mulheres na realização do exame, seja por vergonha, medo ou até mesmo por preceitos religiosos, já que ele relata que a grande maioria de óbitos eram em mulheres portadora do câncer de colo uterino.

O estudo de Melo et al., (2019), ressalta que as mulheres que não realizaram o exame, foi devido sentir vergonha, falta de interesse, falta de tempo, ausência de parceiro, desconforto, medo, crenças e mitos. Santos, conclui que é importante a obtenção da educação continuada dos profissionais que participam da realização do exame e de todas as suas etapas, para que assim os erros sejam corrigidos e que as ações preventivas sejam tomadas para uma melhor qualidade do exame citopatológico 
(Santos \& Ribeiro, 2020). Diante essa revisão percebe-se que a importância do enfermeiro na educação continuada é uma fonte de busca na melhoria do rastreamento do câncer uterino, o qual deve-se explicar a mulher como é realizado, porque realizar e quando realizar o exame.

A vivência da violência, em especial a sexual, pode restringir a busca da consulta ginecológica pela mulher, por medo de retaliação por parte do parceiro, ou ainda por ameaça de desvelamento da violência sofrida. Considerando o impacto da violência na saúde da mulher vítima, tendo em vista isso o serviço de saúde tem um papel importante na promoção de ações de prevenção e rastreamento dos casos de violência contra a mulher, bem como na oferta dos serviços preventivos (Leite, Amorim \& Gigante, 2018). Através das evidências estudadas, é válido ressaltar que os profissionais de saúde devem implementar as ações para alcançar as mulheres, promovendo uma oportunidade de rastreamento para uma assistência de qualidade integral.

\section{Conclusão}

Conclui-se que a necessidade de uma assistência de enfermagem de qualidade deve ser imprescindível para prevenção do câncer de colo de útero, visto que se trata de uma doença que afeta grande parte das mulheres, é necessário cada vez mais de um aprimoramento na assistência, de desenvolver novas visões e estratégias, identificar mais precocemente os fatores de risco, proporcionar atenção individualizada a cada mulher. Proporcionando mais qualidade de vida, tratamento adequado, e melhor relação profissional e paciente.

Observou-se que algumas mulheres ainda apresentam barreiras na realização do exame citopatológico e pouco conhecimento sobre o que reflete a diminuição nas taxas de adesão ao exame. Ainda existe uma escassez de educação na atenção primária, espera-se proporcionar cada vez mais informações, afim de que as mulheres estejam cientes da importância do conhecimento para prevenção do câncer de colo de útero, visto que correm o risco ao longo da vida.

Desse modo, é necessário a busca ativa, visitas em domicílios, para o rastreio de doenças como neoplasias uterinas. Para isso, o Enfermeiro deve ter uma relação baseada no diálogo sincero transpassando confiança, e incentivando a prevenção primaria. Neste sentido, sugere-se mais estudos abordando a temática proposta, afim de buscar cada vez mais conhecimentos com o objetivo de capacitar o profissional da enfermagem para atuar frente a prevenção do câncer de colo do útero.

Neste sentido, sugere-se mais estudos abordando a temática proposta, afim de buscar cada vez mais conhecimentos com o objetivo de capacitar o profissional da enfermagem para atuar frente a prevenção do câncer de colo do útero, para que seja proporcionada um atendimento com assistência mais humanizada, levando a uma boa relação profissional - paciente.

\section{Referências}

Brasil. (2020) Instituto Nacional De Câncer: Atlas da Mortalidade. https://www.inca.gov.br/controle-do-cancer-do-colo-do-utero/conceito-e-magnitude.

Brasil. (2016) Instituto Nacional de Câncer José Alencar Gomes da Silva. Coordenação de Prevenção e Vigilância. Divisão de Detecção Precoce e Apoio à Organização de Rede. Diretrizes brasileiras para o rastreamento do câncer do colo do útero. (2a ed.), INCA.

Brasil.(2016) Instituto Nacional de Câncer José Alencar Gomes da Silva. Coordenação de Prevenção e Vigilância. Divisão de Detecção Precoce e Apoio à Organização de Rede. Diretrizes brasileiras para o rastreamento do câncer do colo do útero. (2a ed.), INCA.

Brasil.(2016) Instituto Nacional De Câncer: Estimativa 2020: Incidência do Câncer no https://www.inca.gov.br/sites/ufu.sti.inca.local/files/media/document/estimativa-2020-incidencia-de-cancer-no-brasil.pdf.

Baia, E.M.; et al. (2018). Dificuldades Enfrentadas pelas Mulheres para Realizar o Exame Papanicolau: revisão integrativa. Revista Nursing. 26 , (1) 75-79.

Brandão, A. M. R., Andrade, F. W. R. de, \& Olivindo, D. D. F. de. (2020). The role of nurses in the family health strategy in the management of women with altered results of colpocytology. Research, Society and Development, 9(10), e5899108962. https://doi.org/10.33448/rsd-v9i10.8962

Barreto, M. L. (2017). Desigualdades em saúde: uma perspectiva global. Ciênc Saúde Colet. 22, 2097-2108.

Catafesta, et al. (2015). Consulta de enfermagem ginecológica na estratégia de saúde da Família. Arq. Ciênc. Saúde. 85-90. 
Ceolin, et al. (2020). Análise do rastreamento do câncer do colo do útero de um município do sul do Brasil. Revista Online de Pesquisa, 406-412.

Cofen. Resolução COFEN No 385/2011. Normatiza a execução, pelo Enfermeiro, da coleta de material para colpocitologia oncótica pelo método de Papanicolau. 2011. http://www. cofen.gov.br/resoluo-cofen-n-3852011_7934. html.

Fernandes, B. M. \& Oliveira, J.L.T. (2017). Intervenções de enfermagem na prevenção do câncer cérvico-uterino: perspectivas das clientes. Revenferm UERJ. 25, 01-06.

Fonsêca, B. J. C.; et al. (2019) Avaliação do Seguimento Clínico de Citopatologia Oncótica em Mulheres na Atenção Primária à Saúde. Rev. brasileira ciência e Saúde. 23,(2),131-140.

Leite, F. M. C., \& Amorim, M. H. C. \& Gigante, D. P. (2018). Implicações das violências contra as mulheres sobre a não realização do exame citopatológico. Revista de Saúde Pública. 52, 1-10.

Luque, J. S; et al. (2016) Social network characteristics and cervical câncer screening among Quechua women in Andean Peru. BMC Public Health. 2-7.

Maciel, N. D.S., et al. (2020). Análise dos resultados do último laudo citopatológico de pacientes com Papanicolau em atraso. Enferm. Foco. 11 ; $129-135$.

Manica, S. T., et al. (2016). Desigualdades socioeconômicas e regionais na cobertura de exames citopatológicos do colo do útero. Ver Gaúcha de Enfermagem. $01-08$.

Melo, E. M. F.; et al. (2019) Cervical cancer: knowledge, attitude and practice on the prevention examination. RevBrasEnferm.

Mendes, K. D. S. \& Silveira, R. C. C. P. \& Galvão, C. M. (2008). Revisão integrativa: Método de pesquisa para a incorporações de evidências na saúde e na enfermagem. Texto Contexto Enferm, Florianópolis, 17, (4), 758-64.

Moher, D. et al. (2015). Principais itens para relatar revisões sistemáticas e meta-análises: A recomendação PRISMA .Epidemio.Serv.Saúde.

Moreira, M. S. \& Santos S. M. C., \& Lima, M. K. C. (2012). Consulta de enfermagem no ambulatório de HIV/AIDS: a percepção dos usuários. Rev Gaúcha Enferm. 33, (3), 52-57.

Oliveira, L. L. D., et al. (2020). Exclusividade na coleta de material para exame de colpocitologia oncótica: percepção dos enfermeiros. Rev. Enferm. UFSM. $10,1-17$.

Rocha, C. B. A., et al. (2019). Insegurança nas ações de controle do câncer de colo uterino: atuação do enfermeiro na Estratégia de Saúde da Família.VerFunCare Online. 1072-1080

Rodrigues, M. \& Moraes, M. D. (2020) Exame citopatológico do colo uterino: descrição dos principais indicadores em um município nordestino. Rev. Ciência Plural. 6, 108-122.

Ronco, G.; et al. (2016). Impact of variations in triage cytology interpretation on human papillomavirus-based cervical screening and implications for screening algorithms. Europe na journal of cancer. P. 148-155.

Santos, M. J. S. \& Ribeiro, A. A. (2020). Estratégias Utilizadas para Melhorar a Qualidade dos Exames Citopatológicos. Ver Bras de Cancerologia.66, 1-7.

Silva, et al. (2017) Screening for cervical cancer in imprisoned women in Brazil. PLOS ONE. 1-15.

Soares, M. B. O. \& Silva, S.R. (2016). Intervenções que favorecem a adesão ao exame de colpocitologia oncótica. RevBrasEnferm. p. $381-91$.

Souza, M. T., et al. (2010). Revisão integrativa: o que é e como fazer. Einsten (São Paulo), v.8, n.1, p.102-106.

Vahabi, M. \& Lofters, A. (2016). Muslim immigrant women's views on cervical cancers creening and HPV self-sampling in Ontario, Canada. BMC Public Health. p. 1-13.

Wang, et al. (2019). Effectiveness of cervical screening after age 60 years according to screening history: nationwide cohort study in sweden. Plos Medicine. $1-17$. 